\title{
Diagnostic value of cerebrospinal fluid tau, neurofilament, and progranulin in definite frontotemporal lobar degeneration
}

\author{
Joery Goossens ${ }^{1,2}$, Maria Bjerke ${ }^{1,2}$, Sara Van Mossevelde $2,3,4,5$, Tobi Van den Bossche ${ }^{2,3,4,5}$, Johan Goeman ${ }^{4}$, \\ Bart De Vil ${ }^{2,6}$, Anne Sieben ${ }^{2,3}$, Jean-Jacques Martin ${ }^{2}$, Patrick Cras ${ }^{2,5,6}$, Peter Paul De Deyn ${ }^{1,2,4}$, \\ Christine Van Broeckhoven ${ }^{2,3}$, Julie van der Zee ${ }^{2,3}$ and Sebastiaan Engelborghs ${ }^{1,2,4^{*}}$ (I)
}

\begin{abstract}
Background: We explored the diagnostic performance of cerebrospinal fluid (CSF) biomarkers in allowing differentiation between frontotemporal lobar degeneration (FTLD) and Alzheimer's disease (AD), as well as between FTLD pathological subtypes.

Methods: CSF levels of routine AD biomarkers (phosphorylated tau ( $p$-tau ${ }_{181}$ ), total tau (t-tau), and amyloid-beta $\left.(A \beta)_{1-42}\right)$ and neurofilament proteins, as well as progranulin levels in both CSF and serum were quantified in definite FTLD $(n=46)$, clinical AD $(n=45)$, and cognitively healthy controls $(n=20)$. FTLD subgroups were defined by genetic carrier status and/ or postmortem neuropathological confirmation (FTLD-TDP: $n=34$, including FTLD-C9orf72: $n=19$ and FTLD-GRN: $n=9$; FTLD-tau: $n=10)$.

Results: GRN mutation carriers had significantly lower progranulin levels compared to other FTLD patients, $A D$, and controls. Both t-tau and $p$-tau ${ }_{181}$ were normal in FTLD patients, even in FTLD-tau. $A \beta_{1-42}$ levels were very variable in FTLD. Neurofilament light chain (Nf-L) was significantly higher in FTLD compared with AD and controls. The reference logistic regression model based on the established AD biomarkers could be improved by the inclusion of CSF Nf-L, which was also important for the differentiation between FTLD and controls. Within the FTLD cohort, no significant differences were found between FTLD-TDP and FTLD-tau, but GRN mutation carriers had higher t-tau and Nf-L levels than C9orf72 mutation carriers and FTLD-tau patients.
\end{abstract}

Conclusions: There is an added value for Nf-L in the differential diagnosis of FTLD. Progranulin levels in CSF depend on mutation status, and GRN mutation carriers seem to be affected by more severe neurodegeneration.

Keywords: Frontotemporal lobar degeneration, Alzheimer's disease, Cerebrospinal fluid, Biomarkers, Differential diagnosis, Tau, Neurofilament, Progranulin

\section{Background}

Frontotemporal lobar degeneration (FTLD) is the primary cause of early-onset dementia after Alzheimer's disease (AD) [1]. Major molecular pathologies underlying FTLD include aggregation of transactive response DNA-binding protein of $43 \mathrm{kDa}$ (TDP-43, FTLD-TDP)

\footnotetext{
* Correspondence: sebastiaan.engelborghs@uantwerpen.be

${ }^{1}$ Reference Center for Biological Markers of Dementia, Laboratory of

Neurochemistry and Behavior, University of Antwerp, Universiteitsplein 1,

2610 Wilrijk, Belgium

${ }^{2}$ Institute Born-Bunge, University of Antwerp, Universiteitsplein 1, 2610 Wilrijk, Belgium

Full list of author information is available at the end of the article
}

or tau (FTLD-tau) [2, 3]. While each molecular pathology is associated with mutations in specific genes (e.g., GRN, C9orf72, MAPT) there is clinical overlap across pathologies and with AD [4-6]. Clinical diagnosis of both dementias is primarily based on exclusion of other diseases, and this results at best in a diagnosis of probable AD or probable FTLD [7-10]. Correct identification of FTLD and its associated pathology is of importance for protein-specific research and treatment (e.g., disease modifiers). Diagnostic accuracy for differentiation between FTLD and AD, as well as between FTLD pathological subtypes, could be increased by quantification of 
disease-specific biochemical markers present in biofluids (cerebrospinal fluid (CSF) and blood) [10, 11].

At present, well-characterized and validated diagnostic markers specific for FTLD pathology do not exist, with the exception of decreased progranulin concentrations in serum or plasma for GRN mutation-related FTLD, an important subgroup of FTLD-TDP [12, 13]. However, progranulin may play a pathophysiological role in the brain as well, independent of GRN mutations [14], and this might be reflected in progranulin levels in CSF which are shown to be mainly brain-derived and regulated independently from levels in the blood $[15,16]$. On the other hand, one previous study has questioned the pathological role of progranulin by showing that CSF progranulin levels could not differentiate between clinically diagnosed groups of AD and FTLD patients [17].

As FTLD can also present with tau-positive inclusions as a primary pathology, the AD CSF biomarkers total tau ( $t$ tau) and hyperphosphorylated tau at threonine 181 (p$\left.\operatorname{tau}_{181}\right)$ are also interesting markers. In theory, an increased concentration of both could be expected since t-tau is an aspecific marker for neurodegeneration and $\mathrm{p}$-tau $\mathrm{t}_{181}$ is a marker of tau pathology [18]. However, in practice, mixed results have been generated over the years, with CSF levels of both biomarkers generally being intermediate in FTLD compared with AD and controls [19]. The combination of $\mathrm{t}$-tau and $\mathrm{p}$-tau 181 with the third AD biomarker amyloidbeta of 42 amino acids $\left(A \beta_{1-42}\right)$ can at least aid in differential dementia diagnosis (reviewed in [20]). It is noteworthy that recent studies have shown that the $\mathrm{p}$ - $\mathrm{tau}_{181} / \mathrm{t}$-tau ratio can be useful in the differentiation of FTLD-tau from FTLD-TDP [21, 22].

Next to pathology-specific biomarkers, other candidates have been proposed. Most promising are neurofilaments which are structural axonal proteins, and their presence in CSF is a marker for neurodegeneration [23]. These proteins might have a more specific role in FTLD-TDP, as TDP-43 interacts with neurofilament light chain (Nf-L) mRNA [24]. It has been reported that both Nf-L and (phosphorylated) neurofilament heavy chain (Nf-H) subunits can be detected in the CSF of FTLD patients, allowing differentiation of FTLD from $\mathrm{AD}$ and controls, and differentiation between FTLD subtypes [25, 26].

This study aimed to explore the performance of proven and candidate CSF biomarkers to improve differential diagnosis of FTLD. A secondary aim was to assess if CSF progranulin levels are dependent on mutation status only or are also related to FTLD pathology.

\section{Methods}

\section{Study cohort}

The study population consisted of definite FTLD patients $(n=46)$, defined by genetic carrier status and/or postmortem neuropathological confirmation [27, 28]. These patients could be subdivided into FTLD-TDP ( $n=34$, including 19 FTLD-C9orf72, 9 FTLD-GRN, 1 FTLD-VCP, and 1 FTLD-TBK1 symptomatic mutation carriers; 19 pathologically confirmed), FTLD-tau $(n=10$, including 1 FTLD-MAPT symptomatic mutation carrier; 9 pathologically confirmed), or FTLD-other $(n=2$, both pathologically confirmed). Clinical diagnosis of definite FTLD patients consisted of the behavioral variant frontotemporal dementia $(n=30)$, $\operatorname{AD}(n=7)$, primary progressive aphasia $(n=3)$, progressive supranuclear palsy $(n=2)$, corticobasal degeneration $(n=1)$, and other types of dementia $(n=3)$. AD patients $(n=45)$ were clinically diagnosed based on IWG-2 criteria, including the AD CSF biomarker panel (pathological cut-offs: $\mathrm{A} \beta_{1-42}<638.5 \mathrm{pg} / \mathrm{mL}, \mathrm{t}$-tau $>296.5 \mathrm{pg} / \mathrm{mL}, \mathrm{p}$-tau ${ }_{181}>$ $56.5 \mathrm{pg} / \mathrm{mL})[10,29]$. Together with extensive clinical follow-up (median 4.9 (range 2.7-7.9) years) this added to the diagnostic certainty. The control group $(n=20)$ consisted of elderly people who had no neurological or psychiatric antecedents and no organic disease involving the central nervous system following extensive clinical examination (patients with polyneuropathy, $n=8$; patients with subjective complaints, $n=12$ ). All CSF samples (full cohorts) and available blood samples (controls, $n=18$; $\mathrm{AD}, n=42$; FTLD, $n=30$ ) were selected from the Institute Born-Bunge (IBB) Biobank, Antwerp, Belgium [30]. Data including gender, Mini-Mental State Examination (MMSE) score, age at time of CSF sampling, age at onset, and age at death (if applicable) were available for the majority of the patients. This study was approved by the ethics committee of the University of Antwerp, Antwerp, Belgium (B300201420405).

\section{Biomarker analysis}

Lumbar puncture (LP), and CSF and blood sampling and handling was performed according to a standardized protocol [30, 31]. All CSF and blood samples were stored at the IBB Biobank in polypropylene vials at $-80{ }^{\circ} \mathrm{C}$ until analysis.

CSF biomarker levels were quantified using commercially available single-analyte enzyme-linked immunosorbent assay (ELISA) kits (one kit lot each), strictly following the manufacturer's instructions (INNOTEST $\beta$-Amyloid(1-42), INNOTEST hTau-Ag, and INNOTEST Phospho-Tau(181P) from Fujirebio Europe, Belgium; Nflight from UmanDiagnostics, IBL International $\mathrm{GmbH}$, Germany; pNF-H V2 from EnCor Biotechnology Inc., USA; human progranulin from Adipogen Inc., Korea). The last kit was also used to quantify serum levels of progranulin. All samples were run in duplicate, blinded for diagnosis. Intra-assay coefficient of variation was below $10 \%$ for all analytes. 


\section{Statistical analysis}

Statistical testing was performed using IBM SPSS Statistics 23, GraphPad Prism 6, and the R package 'pROC' in RStudio (version 1.0.136) [32]. As some variables were not normally distributed and numbers in FTLD subgroups were small, nonparametric analyses were used. Kruskal-Wallis analyses were performed to describe the dementia patient cohorts and compare biomarker levels between groups. Post-hoc analyses included Dunn's correction for multiple comparisons. For pairwise comparisons between FTLD pathological and genetic subgroups, Mann-Whitney $U$ tests were performed. Categorical variables were analyzed with a Chi-square test. Spearman's $\rho$ was calculated to determine correlations. Logistic regression models were generated using a forward selection method of AD biomarkers alone, or of all available single CSF biomarkers. For these analyses, biomarker data were $\log _{10}$-transformed to achieve normality. Receiver operating characteristic (ROC) curve analyses were used to obtain area under the curve (AUC) values with 95\% confidence intervals (CIs) for differentiation between groups [32, 33]. AUC values were compared using DeLong tests. The maximal sum of sensitivity and specificity (maximized Youden's index) was calculated to determine cut-off values for progranulin. For all analyses, $p$ values below 0.05 were considered statistically significant.

\section{Results}

\section{Biomarker results}

Demographic, clinical, and biomarker data for all groups are summarized in Table 1. Two FTLD patients were excluded from the statistical analysis as none of their AD biomarker values could be determined, probably related to preanalytical factors. Biomarker values outside of the assay limits of detection were set to the lowest/highest detection point $\pm 20 \%$, and this value was used in nonparametric statistical analysis (t-tau: 1 FTLD and 5 AD patients; p-tau 181 : 3 FTLD patients; Nf-L: 3 FTLD patients, 2 controls, and 3 AD patients; pNf-H: 9 FTLD, 7 controls, and $8 \mathrm{AD}$ patients). There was no difference in biomarker levels between males and females $(p=0.53)$. Age at LP correlated significantly with Nf-L levels in controls and AD patients, but not in the FTLD group (controls: $\rho=0.837, p<0.001$; AD: $\rho=0.415, p<0.01$; FTLD: $\rho=0.006, p=0.97)$. MMSE score correlated with $\mathrm{A} \beta_{1-42}(0.436, p<0.01)$ and Nf-L $(-0.457, p<0.01)$ levels in AD patients. No notable correlations were found for the different clinical features and biomarker levels in the FTLD group or its subgroups.

\section{Progranulin}

The FTLD group had significantly lower progranulin levels compared with $\mathrm{AD}$ and controls (serum, $p<0.01$;
CSF, $p<0.05)$. This effect was mainly driven by the FTLD-GRN patients, who had the lowest serum and CSF progranulin levels of all groups (Fig. 1); significances disappeared when these patients were excluded. The separation of FTLD-GRN carriers and FTLD patients without GRN mutations was achieved at a cut-off level of $75.3 \mathrm{ng} / \mathrm{mL}$ in serum (91\% sensitivity, 100\% specificity) and at $2.52 \mathrm{ng} / \mathrm{mL}$ in CSF (81\% sensitivity, 88\% specificity).

\section{$A D$ biomarker panel}

There were no significant differences in t-tau or $\mathrm{p}$-tau $\mathrm{t}_{181}$ levels when comparing FTLD patients and controls. Levels of $A \beta_{1-42}$ were normal for the main part of the controls, while covering a wide range from normal to abnormal values in the FTLD group (Fig. 2). Part of this variability could be explained by amyloid- $\beta$ copathology as A2-C1, A2-C2, A3-C2, or A3-C3 scores (representing Thal (A) and CERAD (C) scores in Montine criteria [34-36]) were given in 5/14 of patients with abnormal $\mathrm{A} \beta_{1-42}$ values below the pathological cut-off for $\mathrm{AD}(<$ $638.5 \mathrm{pg} / \mathrm{mL}$ [29]) and in no patients with normal $\mathrm{A} \beta_{1-}$ 42 values above the pathological cut-off. There was no association with age at LP, age at onset, or MMSE score in these patients. Additionally, there was an effect of $A P O E$ \&4 carrier status on $\mathrm{A} \beta_{1-42}$ levels in FTLD ( $p=$ 0.036).

Within the FTLD group, no significant differences were found for $A \beta_{1-42}$ or $\mathrm{p}$-tau $\mathrm{t}_{181}$, but there was a difference in t-tau levels, being significantly higher in FTLDGRN patients (Fig. 2). In these patients t-tau was also significantly higher than in controls $(p<0.05)$. There was no significant difference in $\mathrm{p}$-tau $\mathrm{tan}_{181} / \mathrm{t}$-tau ratio between FTLD-TDP and FTLD-tau groups $(p=0.29)$. While not significantly different from the other FTLD subgroups, $A \beta_{1-42}$ levels in the FTLD-tau group were quite low and did not significantly differ from the AD group $(p=0.20)$.

\section{Neurofilaments}

Nf-L was higher in the FTLD group in comparison with $\mathrm{AD}$ or controls, with post-hoc pairwise comparisons between FTLD and AD or FTLD and controls both being significant $(p<0.01)$. There was no significant difference in pNf-H levels between the diagnostic groups (Fig. 3).

With regard to FTLD subgroups, pNf-H was significantly higher in FTLD-C9orf72 and FTLD-tau compared with FTLD-GRN (both $p<0.05$ ). Conversely, Nf-L was significantly higher in FTLD-GRN compared with FTLD-C9orf72 and FTLD-tau $(p<0.001$ and $p<0.05$, respectively; Fig. 3). The difference between the entire FTLD-TDP subgroup compared to FTLD-tau did not reach significance $(p=0.052)$. While all FTLD subgroups had higher Nf-L levels than controls, this 
Table 1 Demographic information and biomarker data

\begin{tabular}{|c|c|c|c|c|c|c|c|}
\hline & \multirow[b]{3}{*}{ FTLD } & \multicolumn{4}{|l|}{ FTLD subgroups } & \multirow[b]{3}{*}{ Controls } & \multirow[b]{3}{*}{ Alzheimer's disease } \\
\hline & & \multirow[b]{2}{*}{ FTLD-tau } & \multirow[b]{2}{*}{ FTLD-TDP } & \multicolumn{2}{|c|}{ FTLD-TDP subgroups } & & \\
\hline & & & & FTLD-C9orf72 & FTLD-GRN & & \\
\hline Gender, \% male/female ( $n$ ) & $\begin{array}{l}50 / 50 \\
(46)\end{array}$ & $\begin{array}{l}70 / 30 \\
(10)\end{array}$ & $\begin{array}{l}44 / 56 \\
(34)\end{array}$ & $\begin{array}{l}37 / 63 \\
(19)\end{array}$ & $\begin{array}{l}56 / 44 \\
(9)\end{array}$ & $\begin{array}{l}55 / 45 \\
(20)\end{array}$ & $\begin{array}{l}49 / 51 \\
(45)\end{array}$ \\
\hline Age at LP (years) & $\begin{array}{l}63.6 \\
(55.1-71.7)^{a}\end{array}$ & $\begin{array}{l}70.3 \\
(56.2-74.4)\end{array}$ & $\begin{array}{l}63.3 \\
(54.5-71.6)\end{array}$ & $\begin{array}{l}59.0 \\
(53.7-69.5)^{*}\end{array}$ & $\begin{array}{l}67.3 \\
(63.3-71.9)\end{array}$ & $\begin{array}{l}69.4 \\
(61.5-74.7)\end{array}$ & $\begin{array}{l}71.2 \\
(66.7-79.2)\end{array}$ \\
\hline Age at onset (years) & $\begin{array}{l}62.0 \\
(53.8-69.2)^{a}\end{array}$ & $\begin{array}{l}56.0 \\
(54.0-71.0)\end{array}$ & $\begin{array}{l}63.5 \\
(52.8-69.2)\end{array}$ & $\begin{array}{l}56.0 \\
(48.0-67.0)\end{array}$ & $\begin{array}{l}66.0 \\
(61.5-70.0)\end{array}$ & N/A & $\begin{array}{l}69.5 \\
(64.3-75.8)\end{array}$ \\
\hline Age at death (years) & $\begin{array}{l}65.9 \\
(60.2-75.1)\end{array}$ & $\begin{array}{l}74.0 \\
(60.8-76.2)\end{array}$ & $\begin{array}{l}62.9 \\
(57.5-72.5)\end{array}$ & $\begin{array}{l}57.7 \\
(52.3-62.6)^{* \dagger}\end{array}$ & $\begin{array}{l}69.1 \\
(61.1-74.2)\end{array}$ & N/A & N/A \\
\hline APOE \&4 carriers, \% ( $n)$ & $\begin{array}{l}32.4 \\
(37)\end{array}$ & $\begin{array}{l}57.1 \\
(7)\end{array}$ & $\begin{array}{l}27.6 \\
(29)\end{array}$ & $\begin{array}{l}30.8 \\
(13)\end{array}$ & $\begin{array}{l}20.0 \\
(10)\end{array}$ & $\begin{array}{l}33.3 \\
(6)\end{array}$ & $\begin{array}{l}59.5 \\
(42)\end{array}$ \\
\hline MMSE at LP (0-30), (n) & $\begin{array}{l}21(15-25) \\
(29)\end{array}$ & $\begin{array}{l}23(22-27) \\
(5)\end{array}$ & $\begin{array}{l}19(14-25) \\
(23)\end{array}$ & $\begin{array}{l}15(7-21) \\
(10)^{\dagger}\end{array}$ & $\begin{array}{l}23(16-26) \\
(7)\end{array}$ & N/A & $\begin{array}{l}20(15-25) \\
(42)\end{array}$ \\
\hline Serum progranulin (ng/mL) & $\begin{array}{l}95 \\
(60-126)^{a, b}\end{array}$ & $\begin{array}{l}96 \\
(76-167)^{*}\end{array}$ & $\begin{array}{l}95 \\
(61-125)\end{array}$ & $\begin{array}{l}107 \\
(91-125)^{*}\end{array}$ & $\begin{array}{l}48 \\
(39-63)\end{array}$ & $\begin{array}{l}130 \\
(101-175)\end{array}$ & $\begin{array}{l}119 \\
(98-145)\end{array}$ \\
\hline CSF progranulin (ng/mL) & $\begin{array}{l}3.39 \\
(2.29-3.85)^{a}\end{array}$ & $\begin{array}{l}3.79 \\
(2.94-4.23)^{*}\end{array}$ & $\begin{array}{l}3.00 \\
(2.10-3.64)\end{array}$ & $\begin{array}{l}3.21 \\
(2.35-3.74)^{*}\end{array}$ & $\begin{array}{l}1.93 \\
(0.97-2.43)\end{array}$ & $\begin{array}{l}3.61 \\
(2.92-4.50)\end{array}$ & $\begin{array}{l}3.88 \\
(3.22-4.59)\end{array}$ \\
\hline $\operatorname{CSF} A \beta_{1-42}(\mathrm{pg} / \mathrm{mL})$ & $\begin{array}{l}641 \\
(457-858)^{a}\end{array}$ & $\begin{array}{l}543 \\
(438-960)\end{array}$ & $\begin{array}{l}698 \\
(481-862)\end{array}$ & $\begin{array}{l}695 \\
(457-819)\end{array}$ & $\begin{array}{l}708 \\
(578-943)\end{array}$ & $\begin{array}{l}812 \\
(646-1108)\end{array}$ & $\begin{array}{l}509 \\
(372-594)^{b}\end{array}$ \\
\hline CSF t-tau (pg/mL) & $\begin{array}{l}320 \\
(219-420)^{a}\end{array}$ & $\begin{array}{l}331 \\
(197-400)\end{array}$ & $\begin{array}{l}330 \\
(236-464)\end{array}$ & $\begin{array}{l}252 \\
(178-332)^{*}\end{array}$ & $\begin{array}{l}379 \\
(296-559)\end{array}$ & $\begin{array}{l}257 \\
(173-381)\end{array}$ & $\begin{array}{l}627 \\
(429-928)^{b}\end{array}$ \\
\hline CSF p-tau $181(p g / m L)$ & $\begin{array}{l}36.7 \\
(28.3-49.0)^{a}\end{array}$ & $\begin{array}{l}35.5 \\
(26.9-58.8)\end{array}$ & $\begin{array}{l}37.2 \\
(28.8-49.0)\end{array}$ & $\begin{array}{l}33.0 \\
(22.0-49.0)\end{array}$ & $\begin{array}{l}36.0 \\
(30.9-42.5)\end{array}$ & $\begin{array}{l}40.3 \\
(32.9-58.6)\end{array}$ & $\begin{array}{l}80.0 \\
(60.5-105.0)^{b}\end{array}$ \\
\hline CSF p-tau $181 /$ t-tau ratio & $\begin{array}{l}0.117 \\
(0.097-0.149)^{\mathrm{b}}\end{array}$ & $\begin{array}{l}0.144 \\
(0.095-0.152)^{*}\end{array}$ & $\begin{array}{l}0.116 \\
(0.092-0.145)\end{array}$ & $\begin{array}{l}0.142 \\
(0.105-0.164)^{*}\end{array}$ & $\begin{array}{l}0.088 \\
(0.076-0.114)\end{array}$ & $\begin{array}{l}0.176 \\
(0.156-0.197)\end{array}$ & $\begin{array}{l}0.132 \\
(0.104-0.149)^{b}\end{array}$ \\
\hline CSF Nf-L (pg/mL) & $\begin{array}{l}3967 \\
(2556-7148)^{a, b}\end{array}$ & $\begin{array}{l}2508 \\
(1217-6053)^{*}\end{array}$ & $\begin{array}{l}4323 \\
(3367-7358)\end{array}$ & $\begin{array}{l}3446 \\
(2611-4049)^{*}\end{array}$ & $\begin{array}{l}7323 \\
(5432-9097)\end{array}$ & $\begin{array}{l}1136 \\
(547-3984)\end{array}$ & $\begin{array}{l}1597 \\
(1281-2781)\end{array}$ \\
\hline CSF pNf-H (ng/mL) & $\begin{array}{l}0.43 \\
(0.21-1.25)\end{array}$ & $\begin{array}{l}0.54 \\
(0.29-1.45)^{*}\end{array}$ & $\begin{array}{l}0.41 \\
(0.16-0.95)\end{array}$ & $\begin{array}{l}0.45 \\
(0.22-0.95)^{*}\end{array}$ & $\begin{array}{l}0.04 \\
(0.04-0.33)\end{array}$ & $\begin{array}{l}0.33 \\
(0.04-1.36)\end{array}$ & $\begin{array}{l}0.47 \\
(0.19-1.43)\end{array}$ \\
\hline
\end{tabular}

Values are presented as median (interquartile range) unless otherwise indicated

Gender and MMSE were analyzed with a Chi-squared test

Kruskal-Wallis test with post-hoc Dunn's correction was used to compare (full) dementia groups and controls

Significant differences $(p<0.05)$ are indicated: ${ }^{a}$ compared to Alzheimer's disease; ${ }^{b}$ compared to controls

Pairwise Mann-Whitney $U$ tests were used to compare FTLD subgroups

Significant differences $(p<0.05)$ are indicated: * compared to FTLD-GRN; ${ }^{\dagger}$ compared to FTLD-tau

$A \beta$ amyloid-beta, CSF cerebrospinal fluid, FTLD frontotemporal lobar degeneration, $L P$ lumbar puncture, Nf-L neurofilament light chain, MMSE Mini-Mental State

Examination, N/A not applicable, $p N f-H$ phosphorylated neurofilament heavy chain, $p$-tau 181 phosphorylated tau, $t$-tau total tau

difference was only significant for FTLD-GRN and FTLD-TDP (both $p<0.001$ ). The same results were found when comparing the different FTLD subgroups with AD patients, but with an additional significance for FTLD-C9orf72 $(p<0.05)$.

\section{Correlations between biomarkers}

Interestingly, serum and CSF progranulin levels correlated only in the FTLD cohort. Specifically, this correlation was seen in both FTLD-tau ( $\rho=0.900, p<0.05, n=5)$ and FTLD-TDP ( $\rho=0.765, p<0.001, n=22)$, but in the latter group this was limited to FTLD-GRN ( $\rho=0.821, p<$ $0.05, n=7)$ as the correlation was absent in FTLDC9orf72 ( $\rho=0.127, p=0.71, n=11)$. A remarkable correlation was found between the progranulin serum/ progranulin CSF ratio and Nf-L levels in the GRN mutation carriers $(\rho=0.929, p<0.01)$.

Biomarker levels of $\mathrm{p}$-tau 181 and t-tau correlated strongly in controls, $\mathrm{AD}$, and FTLD groups (controls, $\rho=0.701$; AD, $\rho=0.887$; FTLD, $\rho=0.769$; all $p<0.001$ ). Values of pNf-H and Nf-L were significantly correlated in controls and AD groups but not in FTLD (controls, $\rho=0.641$; AD, $\rho=0.346$; both $p<0.05$ ). A correlation could be found for $\mathrm{Nf}-\mathrm{L}$ and the $\mathrm{p}$-tau ${ }_{181} / \mathrm{t}$-tau ratio in all groups between both markers for neurodegeneration.

\section{Diagnostic accuracy}

To differentiate between FTLD and AD, a reference logistic regression model generated with the established AD biomarkers achieved an AUC of 0.942 (95\% CI 0.891-0.981). The only investigated biomarker that 


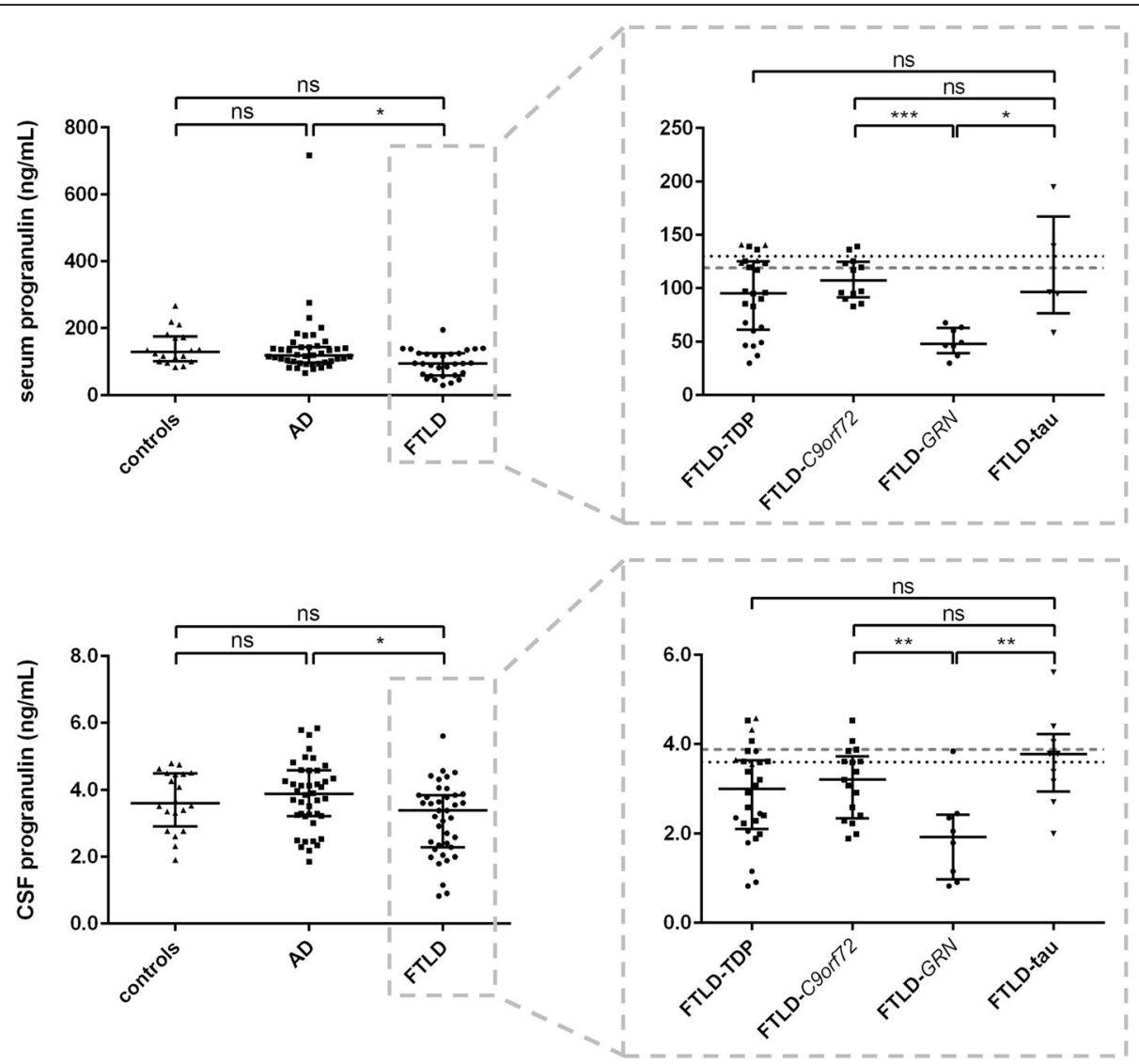

Fig. 1 Dot plots of progranulin levels in all different (sub)groups. Values are presented as median and interquartile range. Left panels compare controls (triangles), Alzheimer's disease (AD; squares), and frontotemporal lobar degeneration (FTLD; circles) dementia groups. Right panels compare FTLD subgroups: FTLD-TDP (triangles, squares (representing FTLD-C9orf72 symptomatic mutation carriers), and circles (representing FTLD-GRN symptomatic mutation carriers)), and FTLD-tau (downward triangles)). The dotted line represents the median control level, and the dashed line represents the median AD level. ${ }^{* * *} p<0.001,{ }^{* *} p<0.01,{ }^{*} p<0.05$. CSF cerebrospinal fluid, ns not significant

added value to this model was $\mathrm{Nf}-\mathrm{L}$, resulting in an AUC of 0.948 (95\% CI $0.900-0.984)$ but this improvement was not significant $(p=0.30)$. The addition of age as a covariate did not change the result of the logistic model (AUC 0.951, 95\% CI 0.908-0.956; $p=0.70$ ).

For the differentiation between FTLD and controls, $\mathrm{A} \beta_{1-42}$ and Nf-L were the best predictors with an AUC of 0.881 (95\% CI 0.761-0.971). The addition of age as a covariate did not significantly alter this model, gaining an AUC of 0.879 (95\% CI 0.759-0.970; $p=0.66$ ).

\section{Discussion}

The lack of biomarkers specific for FTLD is a limiting factor in both clinical and research settings as it severely hampers the diagnostic certainty for this disorder. As such, the aim of this study was to validate both proven and high-ranking candidate CSF biomarkers to improve (differential) diagnosis of FTLD. A major advantage of this study was the availability of a large cohort of definite FTLD patients with genetic and/or neuropathological confirmation. Indeed, discrepancies between publications describing potential biomarkers for FTLD are largely due to the fact that clinical diagnosis is used to define patient groups. Conversely, in publications that used a definite diagnosis, patient groups were usually small, and more data are required to support their findings.

\section{Progranulin}

In this study we confirm the use of decreased progranulin levels in either serum or CSF as a marker for mutation status in FTLD-GRN patients. No evidence was found that progranulin levels in CSF were significantly altered in FTLD patients without GRN mutations.

There are contradicting results about the correlation of blood and CSF progranulin levels in control subjects, being either absent $[37,38]$ or present $[16,17]$. We did not find a significant correlation in either controls or AD patients, but CSF and serum progranulin levels did correlate very strongly in our FTLD-GRN subgroup ( $\rho=0.821$ ). This has only been looked at in one other study so far and, while the correlation was also significant, it was not as strong (Pearson's $r=0.54$ [38]). In our entire cohort 

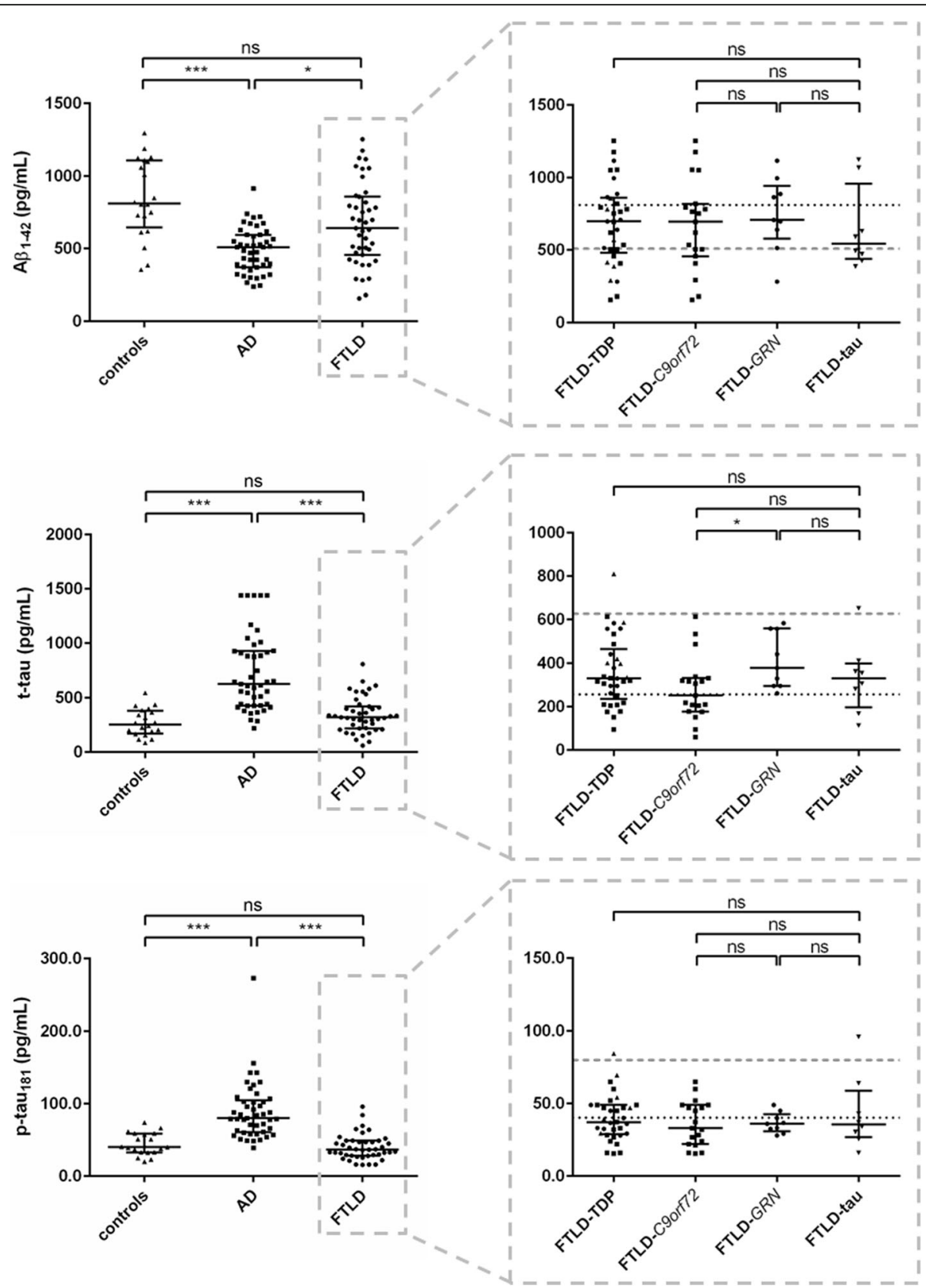

Fig. 2 Dot plots of AD CSF biomarkers in all different (sub)groups. Values are presented as median and interquartile range. Left panels compare controls (triangles), Alzheimer's disease (AD; squares), and frontotemporal lobar degeneration (FTLD; circles) dementia groups. Right panels compare FTLD subgroups: FTLD-TDP (triangles, squares (representing FTLD-C9orf72 symptomatic mutation carriers), and circles (representing FTLD-GRN symptomatic mutation carriers)), and FTLD-tau (downward triangles)). The dotted line represents the median control level, and the dashed line represents the median AD level. ${ }^{* *} p<0.001,{ }^{* *} p<0.01,{ }^{*} p<0.05$. A $\beta$ amyloid-beta, ns not significant, $p$-tau 181 phosphorylated tau, t-tau total tau

with both serum and CSF available $(n=87)$, about $20.7 \%$ of CSF progranulin variability could be explained by serum progranulin variability $\left(\rho^{2}=0.207\right)$. This is considerably higher than previous studies which found that plasma progranulin variability only explained $6.2 \%$ or $13.1 \%$ of CSF progranulin variability, respectively $[16,17]$. However, these studies did not include GRN mutation carriers in their analysis, and when we exclude these subjects the Spearman's $\rho^{2}$ was indeed only 0.099 (i.e., $9.9 \%$ explained) in our entire noncarrier group $(n=80)$. Another difference is the use of the nonparametric Spearman's correlation coefficient in this study instead of the Pearson's correlation coefficient. Using the latter in our entire cohort we found an $r^{2}$ of only 0.105 (i.e., 10.5\% explained). Focusing on the subgroup of GRN mutation carriers with available serum and CSF $(n=7)$, we found that $67.4 \%$ of 

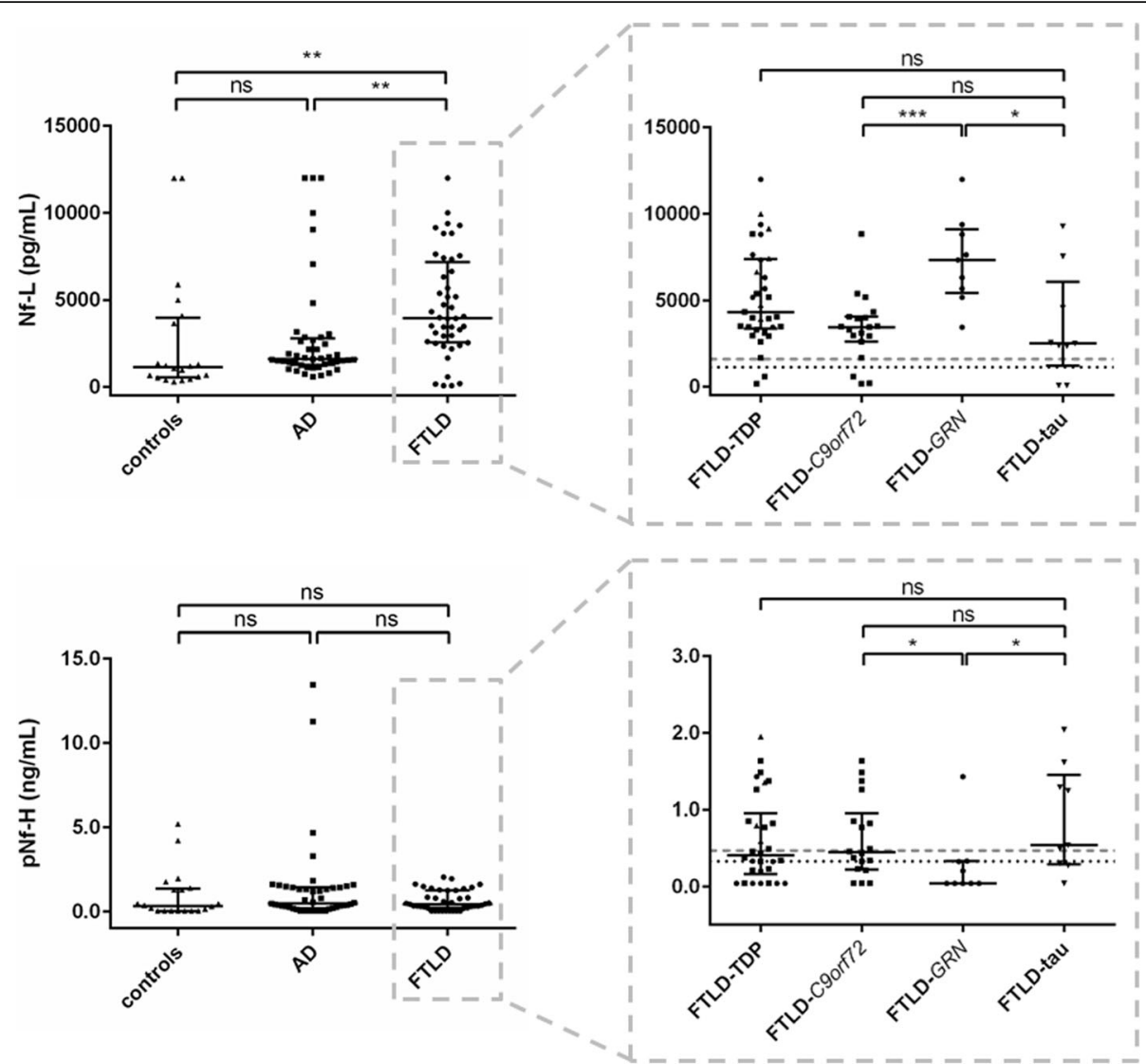

Fig. 3 Dot plots of neurofilament protein levels in all different (sub)groups. Values are presented as median and interquartile range. Left panels compare controls (triangles), Alzheimer's disease (AD; squares), and frontotemporal lobar degeneration (FTLD; circles) dementia groups. Right panels compare FTLD subgroups: FTLD-TDP (triangles, squares (representing FTLD-C9orf72 symptomatic mutation carriers), and circles (representing FTLD-GRN symptomatic mutation carriers)), and FTLD-tau (downward triangles)). The dotted line represents the median control level, and the dashed line represents the median AD level. ${ }^{* *} p<0.001,{ }^{* *} p<0.01,{ }^{*} p<0.05$. Nf-L neurofilament light chain, ns not significant, pNF-H phosphorylated neurofilament heavy chain

CSF progranulin variability could be explained by serum progranulin variability. This is much higher than the $29 \%$ that was reported recently [38]. Our findings indicate that progranulin might thus be assessed in serum instead of CSF, but only for the FTLD-GRN group. As patient cohorts were small in both studies, further investigation of this relationship is definitely warranted.

\section{AD biomarkers}

In the entire FTLD group there was a marked variability in $A \beta_{1-42}$ and $t$-tau levels. The alteration in $A \beta_{1-42}$ was not related to specific FTLD subtypes, although it was more prominent in FTLD-tau. In some patients, a low level of $A \beta_{1-42}$ could be attributed to an $A \beta$ copathology and/or APOE \&4 carrier status, but it might also be connected to individual variation in the $A \beta$ production process [39].

The variability of $\mathrm{t}$-tau was an interesting finding, as the main purpose in using the established $\mathrm{AD}$ markers was to see if tau proteins had an additional value in FTLD subtypes, in particular for FTLD-tau. However, neither t-tau nor $\mathrm{p}$-tau ${ }_{181}$ were significantly different between FTLD-TDP and FTLD-tau, or between FTLD patients and controls. We found that the neuropathological subtypes also had comparable p-tau ${ }_{181} / \mathrm{t}$-tau ratios (although slightly lower in FTLD-TDP than FTLD-tau). This contradicts previous studies that stated that the ratio was significantly lower in FTLD-TDP [21, 22 , 40]. It should be noted that, while they gained significance, differences in $\mathrm{p}$-tau $181 / \mathrm{t}$-tau ratios in these previous studies were also very small. Additionally, there has been much discussion about the (unspecific) pathological changes that would result in a differential p$\operatorname{tau}_{181} / \mathrm{t}$-tau ratio between FTLD-TDP and FTLD-tau [40]. In fact, the ratio has been contradictorily decreased either by lower $\mathrm{p}$-tau ${ }_{181}$ or higher $\mathrm{t}$-tau levels [21, 41]. As such, and together with our data in pathologically confirmed patients, we doubt that the $\mathrm{p}$ - $\mathrm{tau}_{181} / \mathrm{t}$-tau ratio will be valuable in the diagnosis of FTLD subgroups. Interestingly, when looking at genetic subtypes of FTLDTDP, it appears that t-tau is specifically elevated in patients carrying a GRN mutation since their levels were 
significantly higher than those of C9orf72 mutation carriers, at least explaining some of the variability in the FTLD group (see also the paragraph 'Differences within FTLD subtypes' below).

\section{Neurofilament proteins}

With regard to neurofilament levels in controls, $\mathrm{AD}$, and FTLD subjects, there was only a significant increase in Nf-L in FTLD. To evaluate the influence of amyotrophic lateral sclerosis (ALS), which is characterized by high Nf-L levels [42], statistical analysis was performed without FTLD-TDP patients with associated ALS $(n=5)$ which had no effect on the results. In comparison to AD we observed a 2.5 -fold increase in FTLD, which is in concordance with other studies that found a two- to threefold concentration of Nf-L in FTLD compared with $\mathrm{AD}[26,40,43]$. Some older studies did not find a significant difference between the two types of dementias, but these studies had the limitation of assessing clinical FTLD patients only and separating the AD cohort based on age of onset $[25,44]$. We observed more than a threefold increase in Nf-L levels in FTLD compared with controls, which is again in agreement with other studies $[25,26,43,45]$. As for $\mathrm{pNf}-\mathrm{H}$, previous studies with clinical patient cohorts have reported differences in pNf-H levels between FTLD and controls, but differentiation with $\mathrm{AD}$ has not been possible $[25,44,46]$. By confirming the lack of significant differences in definite FTLD patients, we conclude that focus should remain on Nf-L rather than pNf-H.

While we established that Nf-L was higher in FTLD in general, we investigated if there was an association with a specific underlying pathology and found a trend towards increased Nf-L values in FTLD-TDP compared with FTLD-tau $(p=0.052)$. As the same relation has gained significance in other studies $[26,40]$ the small number of FTLD-tau patients in our study likely limited our findings. In the genetic subgroups, a significant difference for Nf-L was found between GRN and C9orf72 mutation carriers (see also the paragraph 'Differences within FTLD subtypes' below).

\section{Combination of biomarkers for differential diagnosis of FTLD}

In this study, the diagnostic accuracy obtained with the established AD biomarkers was used as a reference for comparison with the other investigated biomarkers. It should be noted that, while the use of AD CSF biomarkers is known to increase diagnostic certainty, the clinical diagnosis of $\mathrm{AD}$ patients remains suboptimal which is a limitation of this study and has likely influenced differential diagnostic accuracy. Our results show that only $\mathrm{p}$-tau ${ }_{181}$ and $A \beta_{1-42}$ were necessary to differentiate between AD and FTLD, confirming previous results in definite FTLD patients [47, 48]. The discriminative power could be improved on with the inclusion of Nf-L. While diagnostic accuracies of AD markers on their own and Nf-L on its own have been described previously, only one study performed a joint ROC analysis and also reported an improved diagnostic accuracy when using the combination of $A \beta_{1-42}, p-t_{10 u}$, and Nf-L in comparison with $A \beta_{1-42}$ and $p-t_{181}$ alone [25]. Nf-L also showed added value in the differentiation between FTLD and controls, confirming its usefulness as a marker for FTLD [40, 45].

\section{Differences within FTLD subtypes: genetics and/or neuropathology}

As stated above, both CSF levels of t-tau and Nf-L were markedly higher in FTLD-GRN patients compared with other FTLD subgroups (Table 1). There are limited data available with which to compare these findings, but for Nf-L at least it has been described that levels are significantly higher in FTLD-GRN than in FTLD-C9orf72 and FTLD-MAPT [45]. Regarding tau, one study in contrast reported lower levels of $\mathrm{t}$-tau and $\mathrm{p}$-tau $\mathrm{tan}_{181}$ in FTLD patients with a GRN mutation than FTLD patients without a GRN mutation, although they were not expecting this result [49]. Indeed, there is evidence that FTLD-GRN patients would specifically have more generalized brain atrophy, more white matter lesions, a faster rate of neurodegeneration, and a resulting shorter disease duration [50-53]. Our findings certainly support this notion, as the common feature of both $\mathrm{t}$-tau and Nf-L proteins is that they are markers for general neurodegeneration.

\section{Conclusions}

This study adds to the available biomarker data by validating different markers in an FTLD cohort with definite diagnoses. Nf- $\mathrm{L}$ has an added diagnostic value for the differentiation between FTLD and AD, and between FTLD and controls. The value of progranulin as a biomarker is shown to be limited to those patients with a $G R N$ mutation. Established AD biomarkers are very variable in FTLD and their diagnostic value is based on the exclusion of AD pathology. None of the evaluated biomarkers is able to differentiate between pathological subtypes of FTLD. Also, the relation between CSF and serum levels of progranulin remains ambiguous, but at least appears to be present in GRN mutation carriers. Within this FTLD subgroup, elevated CSF levels of t-tau and Nf-L indicate a more severe neurodegeneration.

\footnotetext{
Abbreviations

AD: Alzheimer's disease; ALS: Amyotrophic lateral sclerosis; AUC: Area under the curve; $A \beta_{1-42}$ : Amyloid-beta of 42 amino acids; $C l$ : Confidence interval; CSF: Cerebrospinal fluid; ELISA: Enzyme-linked immunosorbent assay; FTLD: Frontotemporal lobar degeneration; IBB: Institute Born-Bunge; LP: Lumbar puncture; MMSE: Mini-Mental State Examination; Nf$\mathrm{H}$ : Neurofilament heavy chain; Nf-L: Neurofilament light chain; $\mathrm{p}$-tau ${ }_{181}$ : Tau
} 
protein phosphorylated at threonine 181; ROC: Receiver operating characteristic; TDP-43: Transactive response DNA-binding protein of 43 kDa; t-tau: Total tau protein

\section{Acknowledgements}

The authors acknowledge the technical assistance of N. De Roeck and J. Luyckx for performing CSF analyses.

\section{Funding}

This work was funded in part by the University of Antwerp Research Fund, the Institute Born-Bunge (IBB, www.bornbunge.be), the Flanders Impulse Program on Networks for Dementia Research (VIND), the Agency for Innovation by Science and Technology (IWT, http://www.vlaio.be), and the Research Foundation Flanders (FWO, www.fwo.be). This work has received support from the EU/EFPIA Innovative Medicines Initiative Joint Undertaking (EMIF grant no. 115372). INNOTEST hTau-Ag and INNOTEST Phospho-Tau(181P) ELISA kits were kindly provided by Fujirebio Europe, Belgium, and Nf-light ELISA kits were kindly discounted by IBL International GmbH, Germany.

\section{Availability of data and materials}

The datasets generated and/or analyzed during the current study are available from the corresponding author on reasonable request.

\section{Authors' contributions}

$J G s, M B, J v d Z$, and SE designed the study, interpreted results, and wrote the manuscript draft. SVM, TVdB, JGn, PPDD, and SE contributed to clinical data acquisition and sampling. AS, J-JM, BDV, PC, JvdZ, and CVB contributed to definite patient diagnosis. JGs and MB performed experiments and analyzed compiled data. All authors critically revised the manuscript and approved its contents before submission.

\section{Ethics approval and consent to participate}

This study was approved by the ethics committee of the University of Antwerp, Antwerp, Belgium (B300201420405). Informed consent was obtained from all subjects.

\section{Consent for publication}

Not applicable.

\section{Competing interests}

The authors declare that they have no competing interests.

\section{Publisher's Note}

Springer Nature remains neutral with regard to jurisdictional claims in published maps and institutional affiliations.

\section{Author details}

${ }^{1}$ Reference Center for Biological Markers of Dementia, Laboratory of Neurochemistry and Behavior, University of Antwerp, Universiteitsplein 1, 2610 Wilrijk, Belgium. ${ }^{2}$ Institute Born-Bunge, University of Antwerp, Universiteitsplein 1, 2610 Wilrijk, Belgium. ${ }^{3}$ Neurodegenerative Brain Diseases Group, Center for Molecular Neurology, VIB, Universiteitsplein 1, 2610 Wilrijk, Belgium. ${ }^{4}$ Department of Neurology and Memory Clinic, Hospital Network Antwerp (ZNA) Middelheim and Hoge Beuken, 2660 Antwerpen, Belgium. ${ }^{5}$ Department of Neurology, Antwerp University Hospital, Wilrijkstraat 10, 2650 Edegem, Belgium. 'aboratory of Neurology, Translational Neurosciences, University of Antwerp, Universiteitsplein 1, 2610 Wilrijk, Belgium.

Received: 15 October 2017 Accepted: 1 March 2018

Published online: 20 March 2018

\section{References}

1. Rabinovici GD, Miller BL. Frontotemporal lobar degeneration: epidemiology, pathophysiology, diagnosis and management. CNS Drugs. 2010;24:375-98. https://doi.org/10.2165/11533100-000000000-00000

2. Neumann M, Sampathu DM, Kwong LK, Truax AC, Micsenyi MC, Chou TT, et al. Ubiquitinated TDP-43 in frontotemporal lobar degeneration and amyotrophic lateral sclerosis. Science. 2006;314:130-3. https://doi.org/10. 1126/science. 1134108

3. Taniguchi S, McDonagh AM, Pickering-Brown SM, Umeda Y, Iwatsubo T, Hasegawa M, et al. The neuropathology of frontotemporal lobar degeneration with respect to the cytological and biochemical characteristics of tau protein. Neuropathol Appl Neurobiol. 2004;30:1-18. https://doi.org/10.1046/j.0305-1846.2003.00481.x

4. Graham A, Davies R, Xuereb J, Halliday G, Kril J, Creasey H, et al. Pathologically proven frontotemporal dementia presenting with severe amnesia. Brain. 2005;128:597-605. https://doi.org/10.1093/brain/awh348

5. Alladi S, Xuereb J, Bak T, Nestor P, Knibb J, Patterson K, et al. Focal cortical presentations of Alzheimer's disease. Brain. 2007;130:2636-45. https://doi. org/10.1093/brain/awm213

6. Irwin DJ, Cairns NJ, Grossman M, McMillan CT, Lee EB, Van Deerlin VM, et al. Frontotemporal lobar degeneration: defining phenotypic diversity through personalized medicine. Acta Neuropathol. 2015;129:469-91. https://doi.org/ 10.1007/s00401-014-1380-1

7. Neary D, Snowden J, Gustafson L. Frontotemporal lobar degeneration: a consensus on clinical diagnostic criteria. Neurology. 1998:51:1546-54.

8. Rascovsky K, Hodges JR, Knopman D, Mendez MF, Kramer JH, Neuhaus J, et al. Sensitivity of revised diagnostic criteria for the behavioural variant of frontotemporal dementia. Brain. 2011;134:2456-77. https://doi.org/10.1093/ brain/awr179

9. Gorno-Tempini ML, Hillis AE, Weintraub S, Kertesz A, Mendez M, Cappa SF, et al. Classification of primary progressive aphasia and its variants. Neurology. 2011;76:1006-14. https://doi.org/10.1212/WNL. Ob013e31821103e6

10. Dubois B, Feldman HH, Jacova C, Hampel H, Molinuevo JL, Blennow K, et al. Advancing research diagnostic criteria for Alzheimer's disease: the IWG-2 criteria. Lancet Neurol. 2014;13:614-29. https://doi.org/10.1016/S14744422(14)70090-0

11. Irwin DJ, Trojanowski JQ, Grossman M. Cerebrospinal fluid biomarkers for differentiation of frontotemporal lobar degeneration from Alzheimer's disease. Front Aging Neurosci. 2013;5:6. https://doi.org/10. 3389/fnagi.2013.00006

12. Sleegers K, Brouwers N, Van Damme P, Engelborghs S, Gijselinck I, van der Zee J, et al. Serum biomarker for progranulin-associated frontotemporal lobar degeneration. Ann Neurol. 2009;65:603-9. https:// doi.org/10.1002/ana.21621

13. Ghidoni R, Benussi L, Glionna M, Franzoni M, Binetti G. Low plasma progranulin levels predict progranulin mutations in frontotemporal lobar degeneration. Neurology. 2008;71:1235-9. https://doi.org/10.1212/01.wnl. 0000325058.10218.fC

14. Wilke C, Gillardon F, Deuschle C, Hobert MA, Jansen IE, Metzger FG, et al. Cerebrospinal fluid progranulin, but not serum progranulin, is reduced in GRN-negative frontotemporal dementia. Neurodegener Dis. 2016;17:83-8. https://doi.org/10.1159/000448896

15. Feneberg E, Steinacker $\mathrm{P}$, Volk AE, Weishaupt $\mathrm{JH}$, Wollmer MA, Boxer A, et al Progranulin as a candidate biomarker for therapeutic trial in patients with ALS and FTLD. J Neural Transm. 2016;123:289-96. https://doi.org/10.1007/ s00702-015-1486-1

16. Nicholson AM, Finch NA, Thomas CS, Wojtas A, Rutherford NJ, Mielke MM, et al. Progranulin protein levels are differently regulated in plasma and CSF. Neurology. 2014;82:1871-8. https://doi.org/10.1212/WNL.0000000000000445

17. Morenas-Rodríguez E, Cervera-Carles L, Vilaplana E, Alcolea D, CarmonaIragui $\mathrm{M}$, Dols-Icardo O, et al. Progranulin protein levels in cerebrospinal fluid in primary neurodegenerative dementias. J Alzheimers Dis. 2015:50: 539-46. https://doi.org/10.3233/JAD-150746

18. Blennow K, Hampel H. CSF markers for incipient Alzheimer's disease. Lancet Neurol. 2003;2:605-13. https://doi.org/10.1016/S1474-4422(03)00530-1

19. van Harten AC, Kester MI, Visser P-J, Blankenstein MA, Pijnenburg YA, van der Flier WM, et al. Tau and p-tau as CSF biomarkers in dementia: a meta-analysis. Clin Chem Lab Med. 2011;49:353-66. https://doi.org/10.1515/CCLM.2011.086

20. Rivero-Santana A, Ferreira D, Perestelo-Pérez L, Westman E, Wahlund L-O, Sarría A, et al. Cerebrospinal fluid biomarkers for the differential diagnosis between Alzheimer's disease and frontotemporal lobar degeneration: systematic review, HSROC analysis, and confounding factors. J Alzheimers Dis. 2017;55:625-44. https://doi.org/10.3233/JAD-160366

21. Hu WT, Watts K, Grossman M, Glass J, Lah JJ, Hales C, et al. Reduced CSF ptau181 to tau ratio is a biomarker for FTLD-TDP. Neurology. 2013:81:194552. https://doi.org/10.1212/01.wnl.0000436625.63650.27

22. Borroni B, Benussi A, Archetti $S$, Galimberti D, Parnetti L, Nacmias B, et al. CSF p-tau181/tau ratio as biomarker for TDP pathology in frontotemporal dementia. Amyotroph Lateral Scler Frontotemporal Degener. 2015:16:86-91. https://doi.org/10.3109/21678421.2014.971812 
23. Petzold A. Neurofilament phosphoforms: surrogate markers for axonal injury, degeneration and loss. J Neurol Sci. 2005;233:183-98. https://doi.org/ 10.1016/j.jns.2005.03.015

24. Strong MJ, Volkening K, Hammond R, Yang W, Strong W, Leystra-Lantz C, et al. TDP43 is a human low molecular weight neurofilament (hNFL) mRNAbinding protein. Mol Cell Neurosci. 2007;35:320-7. https://doi.org/10.1016/j. mcn.2007.03.007

25. de Jong D, Jansen RWMM, Pijnenburg YAL, van Geel WJA, Borm GF, Kremer $\mathrm{HPH}$, et al. CSF neurofilament proteins in the differential diagnosis of dementia. J Neurol Neurosurg Psychiatry. 2007;78:936-8. https://doi.org/10. 1136/jnnp.2006.107326

26. Landqvist Waldö M, Frizell Santillo A, Passant U, Zetterberg H, Rosengren L, Nilsson C, et al. Cerebrospinal fluid neurofilament light chain protein levels in subtypes of frontotemporal dementia. BMC Neurol. 2013;13:54. https:// doi.org/10.1186/1471-2377-13-54

27. Mackenzie IRA, Neumann M, Bigio EH, Cairns NJ, Alafuzoff I, Kril J, et al. Nomenclature and nosology for neuropathologic subtypes of frontotemporal lobar degeneration: an update. Acta Neuropathol. 2010;119: 1-4. https://doi.org/10.1007/s00401-009-0612-2

28. Mackenzie IRA, Neumann M, Baborie A, Sampathu DM, Du Plessis D, Jaros E, et al. A harmonized classification system for FTLD-TDP pathology. Acta Neuropathol. 2011;122:111-3. https://doi.org/10.1007/s00401-011-0845-8

29. Van der Mussele S, Fransen E, Struyfs H, Luyckx J, Mariën P, Saerens J, et al. Depression in mild cognitive impairment is associated with progression to Alzheimer's disease: a longitudinal study. J Alzheimers Dis. 2014;42:1239-50. https://doi.org/10.3233/JAD-140405

30. Engelborghs S, De Vreese K, Van de Casteele T, Vanderstichele H, Van Everbroeck B, Cras P, et al. Diagnostic performance of a CSF-biomarker panel in autopsy-confirmed dementia. Neurobiol Aging. 2008;29:1143-59. https://doi.org/10.1016/j.neurobiolaging.2007.02.016

31. Del Campo M, Mollenhauer B, Bertolotto A, Engelborghs S, Hampel H, Simonsen $\mathrm{AH}$, et al. Recommendations to standardize preanalytical confounding factors in Alzheimer's and Parkinson's disease cerebrospinal fluid biomarkers: an update. Biomark Med. 2012;6:419-30. https://doi.org/10. 2217/bmm.12.46

32. Robin X, Turck N, Hainard A, Tiberti N, Lisacek F, Sanchez J-C, et al. pROC: an open-source package for $\mathrm{R}$ and $\mathrm{S}+$ to analyze and compare ROC curves. BMC Bioinformatics. 2011;12:77. https://doi.org/10.1186/1471-2105-12-77

33. Gude JA, Mitchell MS, Ausband DE, Sime CA, Bangs EE. Internal validation of predictive logistic regression models for decision-making in wildlife management. Wildl Biol. 2009;15:352-69. https://doi.org/10.2981/08-057

34. Montine TJ, Phelps CH, Beach TG, Bigio EH, Cairns NJ, Dickson DW, et al. National Institute on Aging-Alzheimer's Association guidelines for the neuropathologic assessment of Alzheimer's disease: a practical approach. Acta Neuropathol. 2012;123:1-11. https://doi.org/10.1007/s00401-011-0910-3

35. Thal DR, Rüb U, Orantes M, Braak H. Phases of Abeta deposition in the human brain and its relevance for the development of AD. Neurology. 2002; 58:1791-800. https://doi.org/10.1212/WNL.58.12.1791

36. Mirra SS, Heyman A, McKeel D, Sumi SM, Crain BJ, Brownlee LM, et al. The Consortium to Establish a Registry for Alzheimer's Disease (CERAD). Part II. Standardization of the neuropathologic assessment of Alzheimer's disease. Neurology. 1991;41:479-86.

37. Wilke C, Gillardon F, Deuschle C, Dubois E, Hobert MA. Müller vom Hagen J, et al. Serum levels of progranulin do not reflect cerebrospinal fluid levels in neurodegenerative disease. Curr Alzheimer Res. 2016;13:654-62. https://doi. org/10.2174/1567205013666160314151247

38. Meeter LHH, Patzke H, Loewen G, Dopper EGP, Pijnenburg YAL, van Minkelen $R$, et al. Progranulin levels in plasma and cerebrospinal fluid in granulin mutation carriers. Dement Geriatr Cogn Dis Extra. 2016;6:330-40. https://doi.org/10.1159/000447738

39. Janelidze $\mathrm{S}$, Zetterberg $\mathrm{H}$, Mattsson $\mathrm{N}$, Palmqvist $\mathrm{S}$, Vanderstichele $\mathrm{H}$, Lindberg $O$, et al. CSF A $442 / A \beta 40$ and $A \beta 42 / A \beta 38$ ratios: better diagnostic markers of Alzheimer disease. Ann Clin Transl Neurol. 2016;3:154-65. https:// doi.org/10.1002/acn3.274

40. Pijnenburg YAL, Verwey NA, van der Flier WM, Scheltens P, Teunissen CE. Discriminative and prognostic potential of cerebrospinal fluid phosphoTau/ tau ratio and neurofilaments for frontotemporal dementia subtypes. Alzheimer's Dement Diagnosis, Assess Dis Monit. 2015;1:505-12. https://doi. org/10.1016/j.dadm.2015.11.001

41. Wilke C, Deuschle C, Rattay TW, Maetzler W, Synofzik M. Total tau is increased, but phosphorylated tau not decreased, in cerebrospinal fluid in amyotrophic lateral sclerosis. Neurobiol Aging. 2015;36:1072-4. https://doi. org/10.1016/j.neurobiolaging.2014.10.019

42. Gaiottino J, Norgren N, Dobson R, Topping J, Nissim A, Malaspina A, et al. Increased neurofilament light chain blood levels in neurodegenerative neurological diseases. PLoS One. 2013;8:e75091. https://doi.org/10.1371/ journal.pone.0075091

43. Scherling CS, Hall T, Berisha F, Klepac K, Karydas A, Coppola G, et al. Cerebrospinal fluid neurofilament concentration reflects disease severity in frontotemporal degeneration. Ann Neurol. 2014;75:116-26. https:/doi.org/ 10.1002/ana.24052

44. Pijnenburg YAL, Janssen JC, Schoonenboom NSM, Petzold A, Mulder C, Stigbrand T, et al. CSF neurofilaments in frontotemporal dementia compared with early onset Alzheimer's disease and controls. Dement Geriatr Cogn Disord. 2007;23:225-30. https://doi.org/10.1159/000099473

45. Meeter LH, Dopper EG, Jiskoot LC, Sanchez-Valle R, Graff C, Benussi L, et al. Neurofilament light chain: a biomarker for genetic frontotemporal dementia. Ann Clin Transl Neurol. 2016;3:623-36. https://doi.org/10.1002/ acn3.325

46. Brettschneider J, Petzold A, Schottle D, Claus A, Riepe M, Tumani H. The neurofilament heavy chain $(\mathrm{NfH})$ in the cerebrospinal fluid diagnosis of Alzheimer's disease. Dement Geriatr Cogn Disord. 2006;21:291-5. https://doi. org/10.1159/000091436

47. Struyfs H, Niemantsverdriet E, Goossens J, Fransen E, Martin J-J, De Deyn PP, et al. Cerebrospinal fluid p-tau181P: biomarker for improved differential dementia diagnosis. Front Neurol. 2015;6:1-8. https://doi.org/10.3389/fneur. 2015.00138

48. Seeburger JL, Holder DJ, Combrinck M, Joachim C, Laterza O, Tanen M, et al. Cerebrospinal fluid biomarkers distinguish postmortem-confirmed Alzheimer's disease from other dementias and healthy controls in the OPTIMA cohort. J Alzheimers Dis. 2015;44:525-39. https://doi.org/10.3233/ JAD-141725

49. Carecchio M, Fenoglio C, Cortini F, Comi C, Benussi L, Ghidoni R, et al. Cerebrospinal fluid biomarkers in progranulin mutations carriers. Alzheimers Dis. 2011;27:781-90. https://doi.org/10.3233/JAD-2011-111046

50. Josephs KA, Ahmed Z, Katsuse O, Parisi JF, Boeve BF, Knopman DS, et al. Neuropathologic features of frontotemporal lobar degeneration with ubiquitin-positive inclusions with progranulin gene (PGRN) mutations. J Neuropathol Exp Neurol. 2007;66:142-51. https://doi.org/10.1097/nen. Ob013e31803020cf

51. Caroppo P, Le Ber I, Camuzat A, Clot F, Naccache L, Lamari F, et al. Extensive white matter involvement in patients with frontotemporal lobar degeneration. JAMA Neurol. 2014;71:1562. https://doi.org/10.1001/ jamaneurol.2014.1316

52. Whitwell $\lrcorner$, Boeve BF, Weigand SD, Senjem ML, Gunter $\lrcorner$, Baker MC, et al. Brain atrophy over time in genetic and sporadic frontotemporal dementia: a study of 198 serial magnetic resonance images. Eur J Neurol. 2015;22:74552. https://doi.org/10.1111/ene.12675

53. Van Mossevelde S, van der Zee J, Gijselinck I, Engelborghs S, Sieben A, Van Langenhove T, et al. Clinical features of TBK1 carriers compared with C9orf72, GRN and non-mutation carriers in a Belgian cohort. Brain. 2016;139: 452-67. https://doi.org/10.1093/brain/awv358

\section{Submit your next manuscript to BioMed Central and we will help you at every step:}

- We accept pre-submission inquiries

- Our selector tool helps you to find the most relevant journal

- We provide round the clock customer support

- Convenient online submission

- Thorough peer review

- Inclusion in PubMed and all major indexing services

- Maximum visibility for your research

Submit your manuscript at www.biomedcentral.com/submit
C Biomed Central 